\title{
Brownian motion with absolute negative mobility
}

\author{
B. Cleuren and C. Van den Broeck \\ Limburgs Universitair Centrum, B-3590 Diepenbeek, Belgium \\ (Received 13 February 2003; published 27 May 2003)
}

\begin{abstract}
An example of Brownian motion with absolute negative mobility is given and analytically studied in a random walk and diffusion model.

DOI: 10.1103/PhysRevE.67.055101

PACS number(s): 02.50.-r, 05.40.-a
\end{abstract}

\section{INTRODUCTION}

Applying an (small) external force on a macroscopic system at equilibrium cannot induce a net motion in this system opposite to the force. Indeed, such a motion would imply that a single heat bath can cyclically transform internal energy into work in violation of the second law [1]. Similarly, a Brownian particle in a system at equilibrium cannot move in a direction opposite to a small external force, as made explicit by the positivity of its mobility $\mu=k_{B} T / D$. When the reference system is not at equilibrium, negative mobility becomes possible. Examples include electrical networks with nonequilibrium elements such as an amplifier leading to negative electric mobility [2], and the more prosaic example of the Brazil nut problem [3] in which a heavy large particle surrounded by lighter and smaller particles can move upwards, against the gravitational force, when the vessel that contains the particles is shaken. For small Brownian entities, negative mobility is by now also well documented in a number of theoretical investigations [4-7], but it has so far not been experimentally observed. The purpose of the present paper is to use a different model for Brownian negative mobility, which has the advantage that it could be easily realized experimentally. Note that the construction from Ref. [7] is presently the subject of an experimental verification, but in this model the reference nonequilibrium state is, in contrast to ours, not a steady state because it requires the application of time oscillatory forcing.

We start by explaining the intuitive idea behind the mode of operation of our system by focusing on a discrete random walk model. A Brownian particle performs a random walk on the three-layer lattice represented in Fig. 1, which is aligned along the $x$ axis. In the upper and lower layers, the motion of the particle is biased along $x$, but in opposite directions $(+x$ and $-x$ direction for upper layer and lower layer, respectively) and with equal amplitude. Transitions between these layers are possible by hopping across the intermediate layer. The latter consists of pockets of three states with access to the upper and lower layers located at the left- and right-hand sides of these pockets, respectively. All the corresponding transitions are unbiased. The overall symmetry thus dictates that the random walker will not acquire a systematic speed along the $x$ axis. Upon application of an external force, however, say along the $+x$ direction, the walker will preferentially reside in the right-hand side of the pockets, facilitating the entry into the lower layer where the motion however is biased into the $-x$ direction. One can expect that when this bias is sufficiently large, a net motion against the direc- tion of the force, hence negative mobility, is observed. We will show below that this is indeed the case. It should also be clear that the above described mechanism is rather general and can be realized by many other similar constructions. One such example with continuous space will be analyzed in full analytic detail in Sec. III, see Fig. 3.

\section{RANDOM WALK MODEL}

The three-layer lattice represented in Fig. 1 is a periodic repetition of a $3 \times 3$ unit cell. The position of a particle in this lattice is thus specified by the following coordinates: the site number $i \in\{1,2,3\}$, the horizontal layer $\sigma \in\{-, 0,+\}$, and the cell number $I \in \mathbb{Z}$. Assuming that the sites have a linear dimension equal to 1 , the corresponding position along the horizontal $x$ axis is $x_{[i, \sigma, I]}=i+3 I$. The probability to find the particle at position $[i, \sigma, I]$ at time $t$ will be denoted by $P_{[i, \sigma, I]}(t)$.

A particle can jump between nearest neighbor sites, as indicated by the arrows. The transition rates are chosen as follows: In the vertical direction, i.e., for allowed transitions between different layers, the rates are equal to $\gamma$. In the horizontal direction, the rates are given as

$$
\begin{array}{cc}
k_{\leftarrow}^{+}=e^{-\beta(B+F) / 2}, & k_{\rightarrow}^{+}=e^{\beta(B+F) / 2} ; \\
k_{\leftarrow}^{0}=e^{-\beta F / 2}, & k_{\rightarrow}^{0}=e^{\beta F / 2} ; \\
k_{\leftarrow}^{-}=e^{-\beta(-B+F) / 2}, & k_{\rightarrow}^{-}=e^{\beta(-B+F) / 2} .
\end{array}
$$

The arrows in the subscripts $\leftarrow$ and $\rightarrow$ denote transitions to the left and to the right, respectively. The factors $B$ and $F$ are the energy differences between neighboring sites along the $x$ axis as a result of the applied bias $(+B, 0$, and $-B$ in the upper, middle, and lower layers, respectively) and the exter-

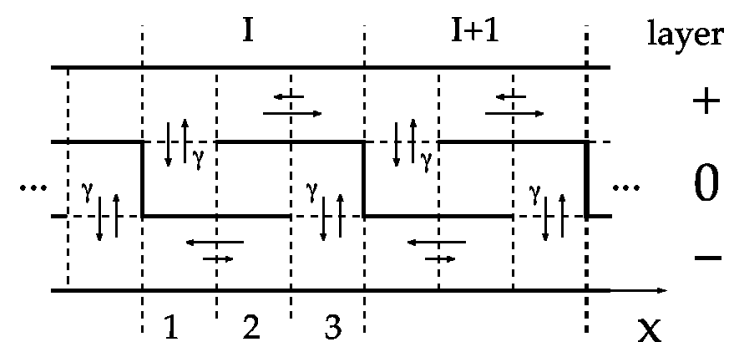

FIG. 1. Representation of the three-layer lattice. Particles can jump between nearest neighbor sites across the dotted lines. 


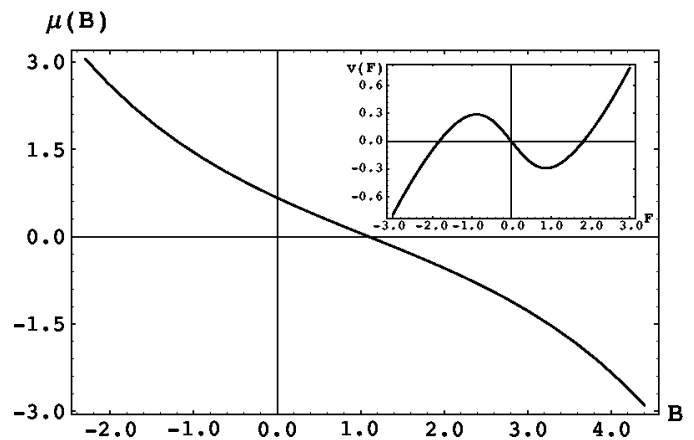

FIG. 2. Plot of the mobility $\mu$ as a function of the bias $B$. The inset shows $v(F)$ for $B=2(\beta=1)$.

nal force (applied in all layers), respectively. The resulting master equation describing the time evolution of $P_{[i, \sigma, I]}(t)$ is reproduced in the Appendix.

Our focus here is on the asymptotic transport properties and, more specifically, on the asymptotic average horizontal velocity $v$ :

$$
v=\lim _{t \rightarrow \infty} \frac{\langle x(t)\rangle}{t}
$$

with $\langle x(t)\rangle=\sum_{i, \sigma, I} x_{[i, \sigma, I]} P_{[i, \sigma, I]}(t)$. Rewriting this as $v$ $=\lim _{t \rightarrow \infty}(\partial / \partial t)\langle x(t)\rangle$ and using the master equation, cf. Eq. (A1), we obtain

$$
v=3\left(k_{\rightarrow}^{+} P_{[3,+]}-k_{\leftarrow}^{+} P_{[1,+]}\right)+3\left(k_{\rightarrow}^{-} P_{[3,-]}-k_{\leftarrow}^{-} P_{[1,-]}\right),
$$

where $P_{[i, \sigma]}=\lim _{t \rightarrow \infty} \sum_{I} P_{[i, \sigma, I]}(t)$ is the steady state probability for a particle to be at any of the sites $i$ in layer $\sigma$. Calculation of the $P_{[i, \sigma]}$ can be reduced to an algebraic problem (see the Appendix), and gives the following result:

$$
\begin{gathered}
P_{[1,0]}=\frac{P_{0} e^{-\beta F}}{1+e^{-\beta F}+e^{\beta F}}, \quad P_{[2,0]}=\frac{P_{0}}{1+e^{-\beta F}+e^{\beta F}}, \\
P_{[3,0]}=\frac{P_{0} e^{\beta F}}{1+e^{-\beta F}+e^{\beta F}}, \quad P_{[i, \pm]}=\frac{1}{3} P_{ \pm} .
\end{gathered}
$$

Here, $P_{\sigma}$ is the reduced probability to be in state $\sigma$ :

$$
P_{ \pm}=\frac{3 e^{\mp \beta F}}{1+4 e^{-\beta F}+4 e^{\beta F}}, \quad P_{0}=\frac{1+e^{-\beta F}+e^{\beta F}}{1+4 e^{-\beta F}+4 e^{\beta F}} .
$$

Combined with Eq. (3), we obtain the final result

$$
v=\frac{3 e^{-\beta(B+F) / 2}\left(e^{3 \beta F}+e^{\beta(B+F)}-e^{\beta(B+2 F)}-1\right)}{4+e^{\beta F}+4 e^{2 \beta F}} .
$$

The corresponding mobility $\mu$ is

$$
\mu=\left.\frac{\partial v(F)}{\partial F}\right|_{F=0}=\frac{1}{3} \beta e^{-\beta B / 2}\left(3-e^{\beta B}\right) .
$$

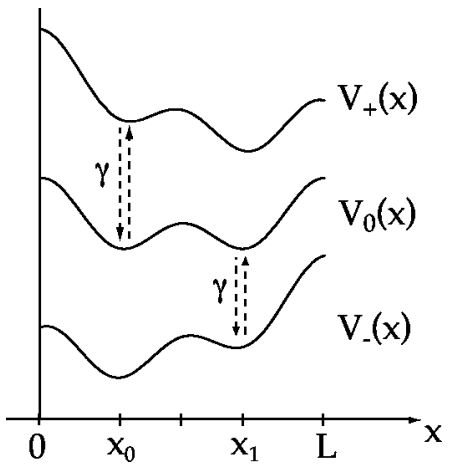

FIG. 3. Schematic representation of the different states and their transitions in the diffusion model. The potential is $V(x)=\cos (x)$ $+\cos (2 x)$.

Figure 2 shows a plot of $\mu$ as a function of the bias $B$. For values of $B$ larger then $\ln (3) / \beta, \mu$ becomes negative. Close to equilibrium, i.e., for small values of $B$, the mobility is positive, as required by the fluctuation dissipation theorem.

\section{DIFFUSION MODEL}

To formulate a spatially continuous analog, we consider an overdamped Brownian particle possessing a discrete degree of freedom $\sigma=-1,0$, or +1 , and moving along the $x$ axis in a one-dimensional potential $V_{\sigma}(x)=V(x)-\sigma x B$, with $V(x)$ spatially periodic with period $L, V(x+L)$ $=V(x)$. Unbiased transitions can take place between the discrete states, but only at specific positions, namely, $x_{0}$ modulo $L$ between $\sigma=0$ and $\sigma=+1$ and $x_{1}$ modulo $L$ between $\sigma$ $=0$ and $\sigma=-1$, cf. Fig. 3. We proceed to find the average velocity along the $x$ axis upon application of an additional force $F$ that is independent of the discrete state. As in the discrete model, the position of a particle is completely determined by the coordinates $x \in[0, L[$, the state variable $\sigma$, and the cell number $I$. The probability to find the particle in state $\sigma$ at position $x+I L$ is $P_{[x, \sigma, I]}(t)$. As before, we define

$$
\begin{gathered}
\langle x(t)\rangle=\sum_{\sigma, I} \int_{0}^{L} d x(x+I L) P_{[x, \sigma, I]}(t), \\
v=\lim _{t \rightarrow \infty} \frac{\partial}{\partial t}\langle x(t)\rangle,
\end{gathered}
$$

and find [see the Appendix, and compare with Eq. (3)] that

$$
v=L \sum_{\sigma} J_{\sigma}
$$

with $J_{\sigma}=\lim _{t \rightarrow \infty} \sum_{I} J_{[x, \sigma, I]}(t)$ the steady state probability current in state $\sigma$, given as

$$
J_{\sigma}=P_{\sigma} \frac{k_{B} T\left[1-e^{-(F+\sigma B) L / k_{B} T}\right]}{\int_{0}^{L} \Phi_{\sigma}(y) d y},
$$




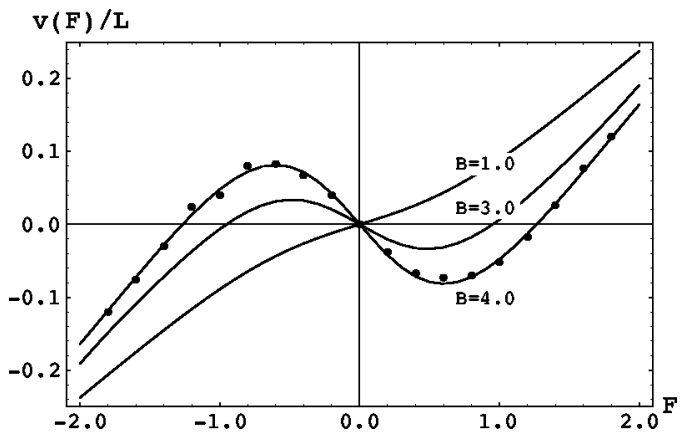

FIG. 4. The average speed $v(F)$ as a function of the applied force $F$ for different values of the bias $B[V(x)=\cos (x)+\cos (2 x)]$. The circles for $B=4$ were obtained by numerical simulations.

$$
\Phi_{\sigma}(x)=\int_{x}^{x+L} d y e^{\left[V_{\sigma}(y)-V_{\sigma}(x)-F(y-x)\right] / k_{B} T} .
$$

$P_{\sigma}$ are the probabilities to be in state $\sigma$ and follow from Eq. (A6) and normalization $P_{-}+P_{0}+P_{+}=1$ :

$$
\begin{aligned}
& P_{-}=\frac{1}{Z}\left[\int_{0}^{L} \Phi_{-}(y) d y\right] \Phi_{0}\left(x_{1}\right) \Phi_{+}\left(x_{0}\right), \\
& P_{0}=\frac{1}{Z} \Phi_{-}\left(x_{1}\right)\left[\int_{0}^{L} \Phi_{0}(y) d y\right] \Phi_{+}\left(x_{0}\right), \\
& P_{+}=\frac{1}{Z} \Phi_{-}\left(x_{1}\right) \Phi_{0}\left(x_{0}\right)\left[\int_{0}^{L} \Phi_{+}(y) d y\right] .
\end{aligned}
$$

The above results are valid for any choice of the potential and the transition points. To reproduce a situation leading to negative mobility, similar in spirit to that of the discrete model, we choose a symmetric potential $V(x)$ with two minima, playing a role akin to the left and right states in the pockets of layer 0 in the discrete model. They are separated from each other on one side by a high maximum, mimicking the absence of transitions from $[1,0, I]$ and $[3,0, I]$ to $[3,0, I$ $-1]$ and $[1,0, I+1]$, respectively, in the discrete model, and a low maximum analogous to the states $[2,0, I]$. It is now also clear as how to choose the points of transition $x_{0}$ and $x_{1}$, namely, at the location of the two minima. For the purpose of illustration, we will use $V(x)=\cos (x)+\cos (2 x)$, and the resulting construction is schematically represented in Fig. 3. The appearance of negative mobility for sufficiently large bias $B$ is illustrated in Fig. 4, showing $v$ as a function of the external force $F$ for different values of the bias $B$.

\section{DISCUSSION}

While there are undoubtedly several direct physical realizations of the basic idea presented here, the most straigthforward being particles suspended in the hydrodynamic flow represented in Fig. 5, we conclude with a more surprising concoction. Consider ellipsoidal particles with a permanent dipole and mass $m$, sedimenting under the influence of a gravitational force in the presence of an electric force acting in the vertical but upward direction. The internal degree of

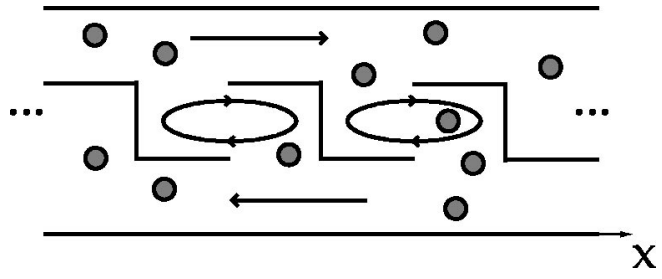

FIG. 5. The arrows represent the hydrodynamic flow in which the suspended particles, represented by the dots, move. Upon application of an external force on these particles, for example, in the $+x$ direction, transitions to the lower layer are more likely, resulting in negative mobility if the flow is sufficiently strong.

freedom analogous to $\sigma$ is here the angle $\theta \in[-\pi, \pi]$ of the particles' dipole axis with the vertical direction (measured clockwise). For a given value of $\theta$, particles acquire an average velocity whose horizontal $x$ component is $v(\theta)$ $=(m g / 2)\left(\mu_{\perp}-\mu_{\|}\right) \sin (2 \theta)$, where $\mu_{\perp}$ and $\mu_{\|}$are the orthogonal $(\theta= \pm \pi / 2)$ and parallel $(\theta=0$ or $\pm \pi)$ mobilities, respectively. The motion is biased to the left and right, respectively, depending on whether $\theta>0$ or $\theta<0$, see Fig. 6 . These $\theta$ values are the analogs of the two oppositely biased states in the basic model. Transitions between different angles occur as a result of rotational diffusion. Because of symmetry, positive and negative $\theta$ values are equally likely and the resulting average drift is zero. The crucial observation is now that the application of an additional electric field along the $x$ axis will favor the internal state $\theta$ that is characterized by a motion induced by the gravitational field in the opposite direction; hence, negative mobility is expected if this effect is larger than the direct response to the electric force.

\section{APPENDIX}

The time evolution of the probability $P_{[i, \sigma, I]}(t)$ is described by the following master equation:

$$
\begin{aligned}
& \partial_{t} P_{[1,+, I]}(t)=-\left(k_{\leftarrow}^{+}+k_{\rightarrow}^{+}\right) P_{[1,+, I]}(t)+k_{\rightarrow}^{+} P_{[3,+, I-1]}(t) \\
& +k_{\leftarrow}^{+} P_{[2,+, I]}(t)+\gamma\left[P_{[1,0, I]}(t)-P_{[1,+, I]}(t)\right], \\
& \partial_{t} P_{[2,+, I]}(t)=-\left(k_{\leftarrow}^{+}+k_{\rightarrow}^{+}\right) P_{[2,+, I]}(t)+k_{\rightarrow}^{+} P_{[1,+, I]}(t) \\
& +k_{\leftarrow}^{+} P_{[3,+, I]}(t),
\end{aligned}
$$

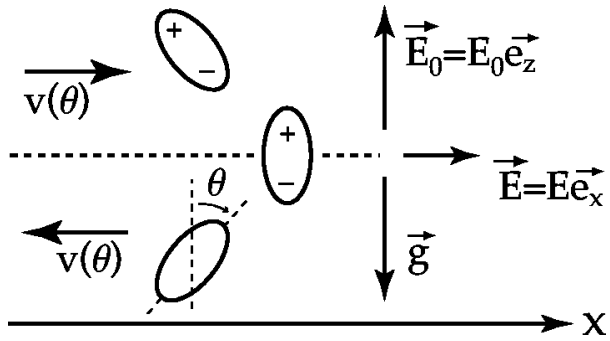

FIG. 6. The application of an additional electric field $\vec{E}$ induces preferential transitions to the configurations that move in the opposite direction. 


$$
\begin{aligned}
\partial_{t} P_{[3,+, I]}(t)= & \left(k_{\leftarrow}^{+}+k_{\rightarrow}^{+}\right) P_{[3,+, I]}(t)+k_{\rightarrow}^{+} P_{[2,+, I]}(t) \\
& +k_{\leftarrow}^{+} P_{[1,+, I+1]}(t), \\
\partial_{t} P_{[1,0, I]}(t)=- & k_{\rightarrow}^{0} P_{[1,0, I]}(t)+k_{\leftarrow}^{0} P_{[2,0, I]}(t)+\gamma\left[P_{[1,+, I]}(t)\right. \\
- & \left.P_{[1,0, I]}(t)\right], \\
\partial_{t} P_{[2,0, I]}(t)= & -\left(k_{\leftarrow}^{0}+k_{\rightarrow}^{0}\right) P_{[2,0, I]}(t)+k_{\rightarrow}^{0} P_{[1,0, I]}(t) \\
& +k_{\leftarrow}^{0} P_{[3,0, I]}(t), \\
\partial_{t} P_{[3,0, I]}(t)=- & k_{\leftarrow}^{0} P_{[3,0, I]}(t)+k_{\rightarrow}^{0} P_{[2,0, I]}(t)+\gamma\left[P_{[3,-, I]}(t)\right. \\
- & \left.P_{[3,0, I]}(t)\right], \\
\partial_{t} P_{[1,-, I]}(t)=- & \left(k_{\leftarrow}^{-}+k_{\rightarrow}^{-}\right) P_{[1,-, I]}(t)+k_{\rightarrow}^{-} P_{[3,-, I-1]}(t) \\
& +k_{\leftarrow}^{-} P_{[2,-, I]}(t), \\
\partial_{t} P_{[2,-, I]}(t)= & -\left(k_{\leftarrow}^{-}+k_{\rightarrow}^{-}\right) P_{[2,-, I]}(t)+k_{\rightarrow}^{-} P_{[1,-, I]}(t) \\
& +k_{\leftarrow}^{-} P_{[3,-, I]}(t), \\
\partial_{t} P_{[3,-, I]}(t)= & -\left(k_{\leftarrow}^{-}+k_{\rightarrow}^{-}\right) P_{[3,-, I]}(t)+k_{\rightarrow}^{-} P_{[2,-, I]}(t) \\
& +k_{\leftarrow}^{-} P_{[1,+, I+1]}(t)+\gamma\left[P_{[3,0, I]}(t)\right. \\
& \left.-P_{[3,-, I]}(t)\right] .
\end{aligned}
$$

Summing both sides of the master equation over $I$ yields a closed set of nine coupled equations for the reduced probabilities $P_{[i, \sigma]}(t)=\Sigma_{I} P_{[i, \sigma, I]}(t)$. The latter quantities approach steady state values in the long time limit $t \rightarrow \infty$. In particular, the total net probability flux between the horizontal layers must vanish, implying

$$
P_{[1,+]}=P_{[1,0]} \text { and } P_{[3,0]}=P_{[3,-]} .
$$

With these conditions, the set of nine equations decouple into three independent sets, one for each layer. Their unique solution is found by including the condition $\Sigma_{i} P_{[i, \sigma]}=P_{\sigma}$. The latter can be determined by using Eq. (A2) and the overall normalization $\Sigma_{\sigma} P_{\sigma}=1$. This procedure can also be re- peated for more complex spatially periodic structures including the calculation of both asymptotic velocity and diffusion coefficient, cf. Ref. [8].

For a continuous space, we proceed in a similar way. The probability $P_{[x, \sigma, I]}(t)$ obeys the master equation

$$
\begin{gathered}
\partial_{t} P_{[x,+, I]}(t)=-\partial_{x} J_{[x,+, I]}(t)+r_{[x,+, I]}(t), \\
\partial_{t} P_{[x, 0, I]}(t)=-\partial_{x} J_{[x, 0, I]}(t)-r_{[x,+, I]}(t)-r_{[x,-, I]}(t), \\
\partial_{t} P_{[x,-, I]}(t)=-\partial_{x} J_{[x,-, I]}(t)+r_{[x,-, I]}(t),
\end{gathered}
$$

where $J_{[x, \sigma, I]}(t)$ is the probability current in state $\sigma$,

$$
J_{[x, \sigma, I]}(t)=-\left[\partial_{x} V_{\sigma}(x)-F+k_{B} T \partial_{x}\right] P_{[x, \sigma, I]}(t)
$$

and $r_{[x, \pm, I]}(t)$ is the net probability flux from state 0 to state \pm at position $x+I L$, as a result of the localized transitions between the different states:

$$
\begin{aligned}
& r_{[x,+, I]}(t)=\gamma \delta\left(x-x_{0}\right)\left[P_{\left[x_{0}, 0, I\right]}(t)-P_{\left[x_{0},+, I\right]}(t)\right], \\
& r_{[x,-, I]}(t)=\gamma \delta\left(x-x_{1}\right)\left[P_{\left[x_{1}, 0, I\right]}(t)-P_{\left[x_{1},-, I\right]}(t)\right] .
\end{aligned}
$$

As before, $P_{[x, \sigma]}=\lim _{t \rightarrow \infty} \Sigma_{I} P_{[x, \sigma, I]}(t)$ is found by summing both sides of the master equation over $I$, and taking the limit $t \rightarrow \infty$. The total net fluxes $r_{[x, \pm]}=\lim _{t \rightarrow \infty} \Sigma_{I} r_{[x, \pm, I]}(t)$ must vanish, so that

$$
P_{\left[x_{0}, 0\right]}=P_{\left[x_{0},+\right]} \text { and } P_{\left[x_{1}, 0\right]}=P_{\left[x_{1},-\right]} .
$$

The result for $P_{[x, \sigma]}$ reads

$$
P_{[x, \sigma]}=P_{\sigma} \frac{\Phi_{\sigma}(x)}{\int_{0}^{L} \Phi_{\sigma}(y) d y},
$$

where $\Phi_{\sigma}(x)$ and $P_{\sigma}$ are given in Eqs. (10) and (11), respectively. Finally, $J_{\sigma}$ is obtained from

$$
J_{\sigma}=-\left[\partial_{x} V_{\sigma}(x)-F+k_{B} T \partial_{x}\right] P_{[x, \sigma]},
$$

leading to the result given in Eq. (10).
[1] H. Callen, Thermodynamics and an Introduction to Thermostatistics (Wiley, New York, 1985).

[2] R. King, Integrated Electronic Circuits and Systems (Van Nostrand Reinhold, Berkshire, 1983).

[3] M.E. Möbius, B.E. Lauerdale, S.R. Nagel, and H.M. Jaeger, Nature (London) 414, 270 (2001).

[4] P. Reimann, R. Kawai, C. Van den Broeck, and P. Hänggi, Europhys. Lett. 45, 545 (1999); J. Buceta, J.M.R. Parrondo, C. Van den Broeck, and J. de la Rubia, Phys. Rev. E 61, 6287
(2000); C. Van den Broeck, I. Bena, P. Reimann, and J. Lehmann, Ann. Phys. 9, 713 (2000).

[5] B. Cleuren and C. Van den Broeck, Europhys. Lett. 54, 1 (2002).

[6] B. Cleuren and C. Van den Broeck, Phys. Rev. E 65, 030101(R) (2002).

[7] R. Eichhorn, P. Reimann, and P. Hänggi, Phys. Rev. Lett. 88, 190601 (2002); Phys. Rev. E 66, 066132 (2002).

[8] I. Claes and C. Van den Broeck, J. Stat. Phys. 70, 1215 (1993). 